\title{
Optimal Motion Control for Connected and Automated Electric Vehicles at Signal-Free Intersections
}

\author{
Xiao Pan, Boli Chen, Simos A. Evangelou and Stelios Timotheou
}

\begin{abstract}
Traffic congestion is one of the major issues for urban traffic networks. The connected and autonomous vehicles (CAV) is an emerging technology that has the potential to address this issue by improving safety, efficiency, and capacity of the transportation system. In this paper, the problem of optimal trajectory planning of battery-electric CAVs in the context of cooperative crossing of an unsignalized intersection is addressed. An optimization-based centralized intersection controller is proposed to find the optimal velocity trajectory of each vehicle so as to minimize electric energy consumption and traffic throughput. Solving the underlying optimization problem for a group of CAVs is not straightforward because of the nonlinear and nonconvex dynamics, especially when the powertrain model is explicitly modelled. In order to ensure a rapid solution search and a unique global optimum, the optimal control problem (OCP) is reformulated via convex modeling techniques. Several simulation case studies show the effectiveness of the proposed approach and the trade-off between energy consumption and traffic throughput is illustrated.
\end{abstract}

\section{INTRODUCTION}

With the aim of addressing the mobility and safety issues related to transportation systems, significant developments of connected and autonomous vehicle (CAV) technology have been achieved during the last decade [1]. With the popularization of CAVs, the cooperative vehicle intersection control has been a popular topic in the research field of intelligent transportation systems. Traditionally, traffic signals are used to regulate flows at intersections even when the vehicles are autonomous. Numerous studies have been conducted to increase the efficiency of traffic light crossing and therefore the fuel economy of the vehicles passing through the intersection [2], [3]. More recently, the advancement of information and communication technologies incentivizes the development of signal-free autonomous intersections, which have the potential of further reducing traffic delays and improving road capacity [4]-[6].

A comprehensive overview of existing autonomous intersection control methods, from centralized to decentralized, can be found in [7], [8]. The centralized approach relies on a single central controller that determines the trajectory of

This work has been supported by the EPSRC Grant EP/N022262/1 and partially funded by the Research Promotion Foundation (Project: CULTURE/BR-NE/0517/14), the European Unions Horizon 2020 research and innovation programme under grant agreement No 739551 (KIOS CoE) and the Government of the Republic of Cyprus through the Directorate General for European Programmes, Coordination and Development.

X. Pan and S. A. Evangelou are with the Dept. of Electrical and Electronic Engineering at Imperial College London, UK (xiao.pan17@ic.ac.uk, s.evangeloulic.ac.uk).

B. Chen is with the Dept. of Electronic and Electrical Engineering at University College London, UK (boli.chen@ucl.ac.uk).

S. Timotheou is with the Dept. of Electrical and Computer Engineering and the KIOS Research and Innovation Center of Excellence, University of Cyprus (timotheou.steliosacy.ac.cy). all the CAVs crossing the intersection. Common centralized approaches are optimization-based with the main objective of increasing the throughput of an intersection [9], which can be achieved by minimizing the travel time. However, the minimization of travel time usually implies high levels of vehicle energy consumption. As such, some effort has focused on the co-optimization of energy consumption and travel time [10], [11], where convex and hierarchical optimization approaches are also utilized to speed up otherwise computationally expensive centralized optimization schemes. Computationally efficient alternatives are the heuristic control strategies [12], [13], which however yield non-optimal control solutions in most cases. There have been numerous other efforts reported in the literature based on decentralized control frameworks, where the velocity of each individual CAV is cooperatively controlled by a local (to each vehicle) controller [14], [15]. In such frameworks, however, a global optimal solution is usually not guaranteed as the decentralized controllers are based only on local information.

Despite the significant progress in studying the traffic management of autonomous vehicles at intersections, the available methods only utilize a highly simplified linear vehicle longitudinal model, in which the energy losses and the powertrain model are neglected. In this context, the energy consumption (objective) is conventionally approximated by the $L^{2}$-norm of the vehicle acceleration, which might not be realistic. The earlier work of the authors [11] proposed a centralized intersection controller with consideration of a realistic longitudinal model involving various resistance losses (such as aerodynamic drag and tire rolling resistance losses) and the possible brake energy recovery to enable more accurate control solutions. In order to make the optimization computational burden manageable, this previous work, however, it still did not take into account the powertrain losses and furthermore it assumed a constant cruising speed for all $\mathrm{CAVs}$ within the center of the intersection. The present paper addresses both of these important deficiencies of autonomous intersection control, while managing to maintain tractability of the associated optimal control problem (OCP). Hence, the contributions of the present work are: 1) a fundamental, and widely used and applicable battery electric powertrain model is integrated into the vehicle dynamics model and thus it permits a more realistic and accurate paradigm of the vehicle energy consumption, 2) the conservative assumption that presumes a constant cruising speed for all CAVs within the center of the intersection is removed and the benefit of this in terms of overall energy and time consumption is verified by numerical examples, and 3) with the additional complexities introduced by 1) and 2), the convexity of the resulting OCP is preserved owing to the employed space- 
domain modeling approach and a non-conservative convex formulation of the electric powertrain. Hence, the overall enhanced centralized intersection problem can be solved to optimality very efficiently.

The remainder of this paper is organized as follows. Section II introduces the intersection crossing problem, along with the electric powertrain model of the CAVs. The intersection problem is formulated as a convex optimization in Section III. Numerical examples are presented in Section IV. Concluding remarks are given in Section V.

\section{Problem Statement}

Fig. 1 shows a two-phase four-approach signal-free intersection with two lanes per road studied in this work. As it can be seen, the center of the intersection is the

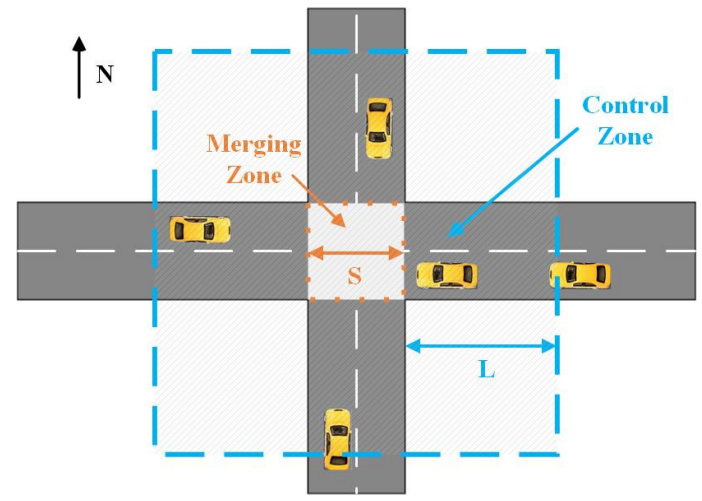

Fig. 1. Scheme of autonomous intersection crossing with connected and autonomous vehicles.

Merging Zone (MZ), where vehicles from different directions merge together, and therefore, it is the area of potential lateral collision of vehicles. Before entering the MZ, CAVs intending to cross the intersection will first enter a Control Zone (CZ), in which the motion of each CAV is fully controlled by an Intersection Controller (IC). Both the MZ and $\mathrm{CZ}$ are considered to be squares of sides $S$ and $2 L+S$, respectively, with $L>S$. The IC determines the trajectories of all the CAVs within the $\mathrm{CZ}$ in a centralized manner to achieve safe intersection crossing with optimized objectives related to the total energy consumption and the total travel time. In this work, it is assumed the IC has no delay and error in communication with the CAVs and the First-In-First-Out (FIFO) policy is imposed to regulate all the CAVs to enter and leave the $\mathrm{MZ}$ in the same order they arrive at the $\mathrm{CZ}$. For simplicity, it is assumed the CAVs studied in this work maintain their initial directions throughout the $\mathrm{MZ}$ and $\mathrm{CZ}$.

For the sake of further discussion, let us denote $N \in \mathbb{N}$ the total number of CAVs arriving at the $\mathrm{CZ}$ within a predefined time-window $T$ and $\mathcal{N}=\{1,2, \ldots, N\} \in \mathbb{Z}^{N}$ the set to designate the order in which the vehicles enter the CZ. In this context, given an arbitrary CAV $i$, the rest of the CAVs can be categorized into the following three subsets of $\mathcal{N}$ : 1) $\mathcal{L}_{i}$ collects vehicles traveling in the same direction as the $i$ th vehicle; 2) $\mathcal{O}_{i}$ collects vehicles traveling in the opposite direction to the $i$ th vehicle; 3) $\mathcal{C}_{i}$ collects vehicles traveling in the perpendicular directions to the $i$ th vehicle.

All the CAVs in this work are considered as identical battery electric vehicles (BEVs) governed by the longitudinal dynamics:

$$
\frac{d}{d t} v_{i}(t)=\frac{F_{w, i}(t)-F_{b, i}(t)-F_{r}-F_{d, i}(t)}{m}, i \in \mathcal{N},
$$

where $v_{i}(t)$ is the linear (forward) velocity of the $i$ th CAV, $m$ is the vehicle mass, $F_{w, i}(t)=\frac{g_{r}}{r_{w}} T_{i}$ is the traction force, $g_{r}$ and $r_{w}$ are the transmission gear ratio and the wheel radius respectively, $T_{i}$ corresponds to the electric motor torque, $F_{b, i}(t)$ denotes the mechanical brake force, and $F_{r}=f_{r} m g$ and $F_{d, i}(t)=f_{d} v_{i}^{2}(t)$ are the resistance forces of rolling and air drag respectively, in which $f_{r}$ and $f_{d}$ are the coefficients of rolling and air drag resistances respectively. The velocity and the motor torque of each CAV are bounded by:

$$
\begin{aligned}
& v_{\min } \leq v_{i}(t) \leq v_{\max } \\
& T_{\min } \leq T_{i}(t) \leq T_{\max }
\end{aligned}
$$

where the speed limits $v_{\max }$ and $v_{\min }$ are set depending on the infrastructure requirements and traffic regulations [10], and the motor torque limits $T_{\max }$ and $T_{\min }$ are specified later in Fig. 2 and in Table I.

It is reasonable to consider the control horizon of the IC is $[0, L+S]$ rather than the whole $\mathrm{CZ}$ as the remaining segment $[L+S, 2 L+S]$ can be integrated into the next intersections with properly sized CZs. Since the travel distance for each CAV is fixed, the problem can be easily formulated in the space domain with an alternative independent variable, the traveled distance, $s$, by a transformation of (1) from the time to the space domain via $\frac{d}{d s}=\frac{1}{v_{i}} \frac{d}{d t}$. Instead of the variable speed $v_{i}(t)$ of the $i$ th vehicle, it is convenient to use the kinetic energy $E_{i}(s)=\frac{1}{2} m v_{i}^{2}(s)$ in the space domain [16]. Therefore, the associated vehicle longitudinal dynamics (1) for all $s \in[0, L+S]$ are governed by:

$$
\frac{d}{d s} E_{i}(s)=F_{w, i}(s)-F_{b, i}(s)-F_{r}-2 \frac{f_{d}}{m} E_{i}(s), i \in \mathcal{N} .
$$

As the variable time $t$ is substituted after domain transformation, it is redefined as a system dynamics state for each vehicle $i$ in the space domain:

$$
\frac{d}{d s} t_{i}(s)=\frac{1}{\sqrt{2 E_{i}(s) / m}}, \quad i \in \mathcal{N} .
$$

The assumption made in previous work [10], [11], [15] that the CAVs follow constant speeds within the MZ is removed in the present work. Instead, the proposed framework allows the velocity trajectories of all CAVs to be fully optimized throughout the mission (over the horizon $L+S$ ). As such, a globally optimal solution can be found rather than a suboptimal solution formed by optimized speed profiles over $[0, L]$ and periods of cruising at non-optimal speeds. The benefit of removing the constant velocity requirement inside the MZ in terms of the energy and travel time saving will be shown in the simulation Section IV (see Fig. 8).

For obvious safety reasons, it is obligatory for the CAVs to avoid rear-end and lateral collisions at all times. The following constraint is imposed to avoid rear-end collisions:

$$
t_{i}(s)-t_{k}(s) \geq \frac{v_{i}(s)-v_{k}(s)}{\left|a_{\min }\right|}, k \in \mathcal{L}_{i}, i \in \mathcal{N},
$$

where $k$ denotes the index of the vehicle in front of the $i$ th 
$\mathrm{CAV}$, and the right hand side of the inequality represents the time-to-collision (TTC) [17] with $a_{\min }$ the maximum available deceleration during emergency braking subject to tire friction limits. Note that the TTC constraint (5) is active only when $v_{i}(s)-v_{k}(s)>0$, and therefore, it is less conservative compared to a nominal time gap as used in [11].

To avoid lateral collisions in the MZ, the following constraint needs to be satisfied for any $\operatorname{CAV} j \in \mathcal{C}_{i}$ that enters the $\mathrm{CZ}$ after $\mathrm{CAV} i$ :

$$
t_{i}(L+S) \leq t_{j}(L), i \in \mathcal{N}, j \in \mathcal{C}_{i}, j>i,
$$

which guarantees that that vehicle $j$ enters the MZ only after vehicle $i$ has left the MZ. Finally, for any CAV $h \in \mathcal{O}_{i}$ the following constraints are imposed:

$$
\begin{aligned}
t_{i}(L)<t_{h}(L), t_{i}(L+S) & <t_{h}(L+S), \\
& i \in \mathcal{N}, \quad h \in \mathcal{O}_{i}, h>i
\end{aligned}
$$

which allows multiple CAVs in the MZ at the same time.

The following assumption is imposed to validate the FIFO priority model:

Assumption 1: The entry times of all CAVs at the $\mathrm{CZ}$ are different, such that $t_{i}(0) \neq t_{j}(0), i \neq j, i, j \in\{1,2, \cdots, N\}$.

Thus, the FIFO policy can be guaranteed by satisfying (5), (6), (7) and Assumption 1. To guarantee all CAVs arriving at the CZ have feasible initial states and initial control inputs, a further assumption is proposed as follows:

Assumption 2: For each CAV $i$, the constraints (2) and (5) are inactive at $t_{i}(0)$.

Since all CAVs in this work are of BEV powertrain architecture, the energy consumption of a CAV is evaluated by the energy cost in its battery. This architecture enables electrochemical power to be absorbed/delivered by the battery to save energy from braking. Such a property may turn out to be beneficial in practice as the maneuvers of CAVs will be regulated and optimized by the IC. The powertrain connected to the battery contains a DC/DC converter, an electric motor and a transmission set, where both the converter and the transmission are simply modelled of constant efficiency values 0.96 , and the efficiency of the motor is modelled as a static efficiency map [18], illustrated in Fig. 2. According to

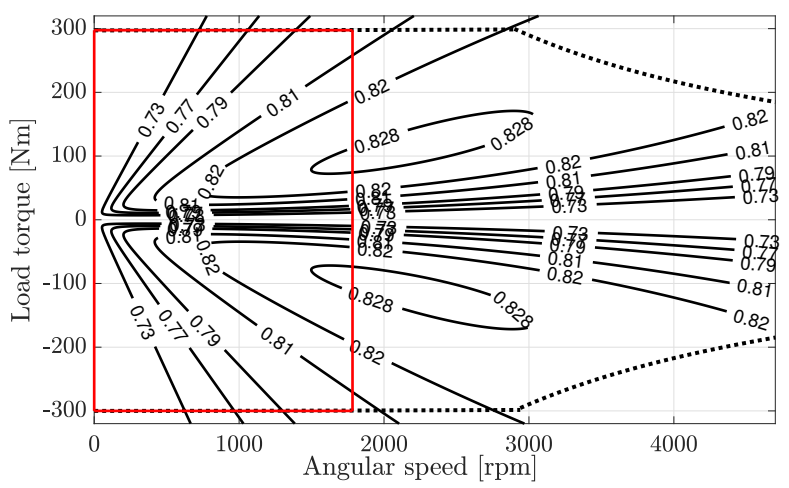

Fig. 2. Efficiency map of the electric motor (positive torque indicates battery discharging and negative torque represents battery charging) and operational bounds (dotted lines) for the reversible motor. The area surrounded by red rectangle denotes the operation region for the feasible vehicle speed specified by (2a) with $v_{\min }$ and $v_{\max }$ defined in Table I.

established literature, the battery power can be represented as a quadratic function of motor torque, given by [19]:

$$
P_{b, i}(s)=b_{1} T_{i}(s) \omega_{i}(s)+b_{2} T_{i}^{2}(s), i \in \mathcal{N},
$$

where $b_{1}$ and $b_{2}$ are fitting parameters, and $\omega_{i}(s)=\frac{g_{r}}{r_{w}} v_{i}(s)$ is the angular speed of the electric motor. By applying (4), the total battery energy usage of all the CAV can be found by integration in the space domain as follows:

$$
J_{b}=\sum_{i}^{N} \int_{0}^{L+S} \frac{P_{b, i}(s)}{\sqrt{2 E_{i}(s) / m}} d s .
$$

\section{Optimal Control Problem Formulation}

The present work proposes optimal control strategies for energy and time management of all electric CAVs crossing a signal-free intersection, subjected to constraints related to vehicle physical limits and safety regulations. The multiobjective cost function of both travel time and battery usage to be minimized is defined as follows:

$$
J=W_{1} \cdot J_{t}+W_{2} \cdot J_{b},
$$

where $J_{t}=\sum_{i=1}^{N}\left(t_{i}(L+S)-t_{i}(0)\right)$ is the total travel time of all the CAVs. Due to the complexity introduced in (10) by the BEV powertrain architecture through the term $J_{b}$, this section formulates the autonomous intersection problem as a convex OCP by certain approximations. These approximations are made under the condition that the approximated problem is conservative and feasible to the original problem.

To convexify the nonlinearity in the state dynamics of the travel time (4), a new control variable $\zeta_{i}(s)$ is introduced:

$$
\begin{aligned}
& \frac{d}{d s} t_{i}(s)=\zeta_{i}(s), \\
& \zeta_{i}(s) \geq \frac{1}{\sqrt{2 E_{i}(s) / m}}, \quad i \in \mathcal{N}
\end{aligned}
$$

such that the nonlinear constraint (4) is replaced by a linear differential equation (11a) and a convex inequality constraint (11b). Since the total travel time objective is minimized in (10), the new input $\zeta_{i}(s)$ finds at all times its minimum boundaries as solutions, which guarantees indirectly the feasibility and conservativeness of the convex solutions. Further verification of the validity of (11b) is performed later in the simulation results (see Fig. 9).

For the TTC constraint (5), after replacing $v_{i}(s)$ with $E_{i}(s)$ the $v_{i}(s)$ term on the right hand side of the inequality becomes non-convex. Thus, a linear approximation is performed between the kinetic energy and the velocity, $v_{i}(s)=\sqrt{2 E_{i}(s) / m}$, as follows:

$$
f\left(E_{i}(s)\right)=a_{0}+a_{1} E_{i}(s), \quad \forall E_{i}(s) \in\left[E_{\min }, E_{\max }\right],
$$

where $f\left(E_{i}(s)\right)$ represents the approximated velocity, and $a_{0}$ and $a_{1}$ are obtained through a constrained least-squares optimization for all $E_{\min } \leq E_{i} \leq E_{\max }$ :

$$
\begin{array}{ll} 
& \min _{a_{0}, a_{1}}\left[f\left(E_{i}\right)-\sqrt{2 E_{i} / m}\right]^{2} \\
\text { s.t.: } & f\left(E_{i}\right)>0, \\
& f\left(E_{i}\right)-\sqrt{\left.2 E_{i}\right) / m} \geq 0,
\end{array}
$$

This optimization (13) guarantees that the linear regression (12) is tangentially fitted to the original relationship, which 
ensures the conservativeness and feasibility of the linear approximation, as shown in Fig. 3. Thus, the TTC constraint

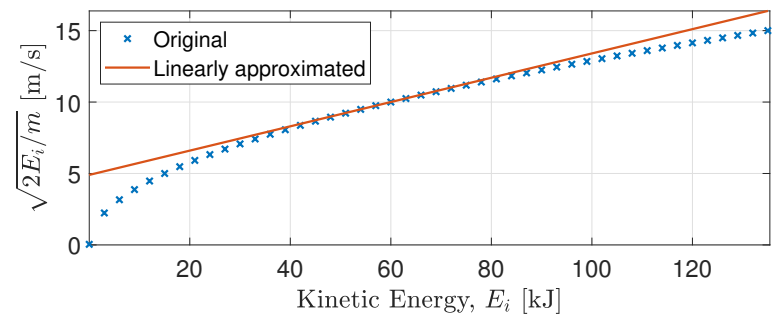

Fig. 3. Linearly approximated relationship between kinetic energy and velocity with R-square fit of $92.27 \%$.

(5) becomes a convex inequality for all $s \in[0, L+S]$ :

$$
t_{i}(s)-t_{k}(s) \geq \frac{a_{0}+a_{1} E_{i}(s)-\sqrt{2 E_{k}(s) / m}}{\left|a_{\min }\right|}, k \in \mathcal{L}_{i},
$$

which is a relaxed and conservative constraint with a larger tolerance on the car-following safety distance, particularly during the low and high speed ranges under the junction speed limit conditions $v_{\min }$ and $v_{\max }$ specified in Table. I.

The battery energy usage defined in (9) is also a nonconvex function, and an approximated battery power $\tilde{P}_{b, i}(s)$ based on a quadratic equation is proposed for OCP convexification, as follows:

$$
\tilde{P}_{b, i}(s)=\tilde{b}_{1} T_{i}^{2}(s) \omega_{i}(s)+\tilde{b}_{2} T_{i}(s) \omega_{i}(s)+\tilde{b}_{3} \omega_{i}(s),
$$

where $\tilde{b}_{1}, \tilde{b}_{2}$ and $\tilde{b}_{3}$ are fitting parameters, with the approximation result having an R-square fit of $99.53 \%$ (see Fig. 4). The expression 15 not only does it help with OCP

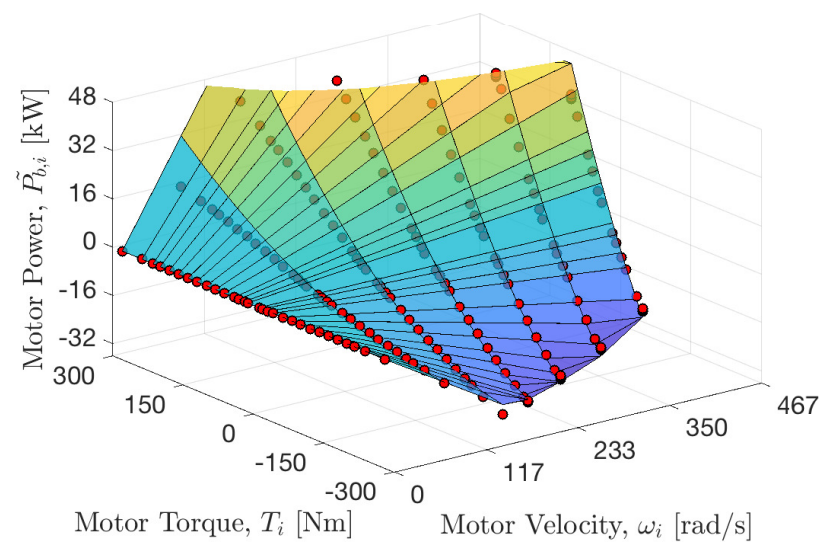

Fig. 4. Motor power $\tilde{P}_{b, i}$ approximated model (15) (red dots denote the motor power $P_{b, i}$ calculated by (8)).

convexification but it also results in better accuracy than the electric motor model fitted in [19].

By substituting (15) into (9), the battery energy usage integral can be rewritten in a convex form:

$$
\tilde{J}_{b}=\frac{g_{r}}{r_{w}} \sum_{i}^{N} \int_{0}^{L+S} \tilde{b}_{1} T_{i}^{2}(s)+\tilde{b}_{2} T_{i}(s)+\tilde{b}_{3} d s .
$$

Thus, the convex OCP for the autonomous intersection problem can be formulated as follows: the system dynamics collect (3) and (11a) as (17b), inequality constraints (17c) collect (2), (6), (11b), and (14), and boundary constraints $(17 \mathrm{~d})$ contain states initial conditions $\left(v_{i}(0), t_{i}(0)\right)$ :

$$
\begin{array}{ll} 
& \min _{\tilde{\boldsymbol{u}}} W_{1} \cdot J_{t}+W_{2} \cdot \tilde{J}_{b} \\
\text { s.t.: } & \frac{d}{d s} \tilde{\boldsymbol{x}}(s)=f(\tilde{\boldsymbol{x}}(s), \tilde{\boldsymbol{u}}(s)), \\
& \Psi(\tilde{\boldsymbol{x}}(s), \tilde{\boldsymbol{u}}(s)) \leq 0, \\
& \boldsymbol{b}(\tilde{\boldsymbol{x}}(0), \tilde{\boldsymbol{x}}(L+S))=0,
\end{array}
$$

and the system state $\tilde{\boldsymbol{x}}$ and inputs $\tilde{\boldsymbol{u}}$ of the convex OCP are

$$
\begin{aligned}
\tilde{\mathbf{x}} & =\left[E_{1}, E_{2}, \ldots, E_{N}, t_{1}, t_{2}, \ldots, t_{N}\right], \\
\tilde{\mathbf{u}} & =\left[T_{1}, T_{2}, \ldots, T_{N}, F_{b, 1}, F_{b, 2}, \ldots, F_{b, N}, \zeta_{1}, \zeta_{2}, \ldots, \zeta_{N}\right] .
\end{aligned}
$$

\section{NUMERICAL RESULTS}

The performance of the proposed scheme is evaluated in this Section. The parameters of the intersection are $L=245 \mathrm{~m}$ and $S=35 \mathrm{~m}$. To streamline the formulation and to make it easy to compare solutions in different cases, it is assumed that all the CAVs leave the MZ at the same terminal speed $v_{i}(L+S)=\bar{v}=10 \mathrm{~m} / \mathrm{s}$; this condition is introduced to the OCP as an additional boundary condition, similarly to (17d), although it is not a general requirement of the proposed scheme and it is applied here merely to enable meaningful comparisons. The characteristic parameters of the battery electric CAVs considered in this work are presented in Table I. Note that the lower bound of the velocity is

TABLE I

Electric Vehicle Model Main Parameters

\begin{tabular}{lcl}
\hline \hline symbol & value & description \\
\hline$m$ & $1200 \mathrm{~kg}$ & vehicle mass \\
$r_{w}$ & $0.3 \mathrm{~m}$ & wheel radius \\
$g_{r}$ & 3.5 & transmission gear ratio \\
$f_{r}$ & 0.01 & rolling resistance coefficient \\
$f_{d}$ & 0.47 & air drag resistance coefficient \\
$v_{\min }$ & $0.1 \mathrm{~m} / \mathrm{s}$ & minimum velocity \\
$v_{\max }$ & $15 \mathrm{~m} / \mathrm{s}$ & maximum velocity \\
$T_{\max } / \min$ & $\pm 300 \mathrm{Nm}$ & motor torque limits \\
$a_{\min }$ & $-6.5 \mathrm{~m} / \mathrm{s}^{2}$ & vehicle maximum deceleration \\
\hline
\end{tabular}

set to a small positive value, rather than zero, in order to guarantee at all times the feasibility of the travel time state dynamics (4) in the space domain. It can be inferred from the vehicle limit $v_{\max }$ that the motor for the studied intersection problem is operated under $1800 \mathrm{rpm}$, which enables fixed torque boundaries for the problem formulation (see Fig. 2). Without loss of generality, the arrival times and initial speeds of all the CAVs are generated randomly. In particular, the initial speeds $v_{i}(0)$ follow a uniform distribution within $\left[v_{\min }, v_{\max }\right]$, while the arrival times $t_{i}(0)$ are modelled using Poisson processes, under the constraints of Assumptions 1 and 2. The evaluation of the proposed scheme (convex OCP (17)) is performed in the Matlab environment using the convex optimization tool CVX with MOSEK solver [20] on an Intel i5 $2.9 \mathrm{GHz} \mathrm{CPU}$ with $8 \mathrm{~GB}$ of memory.

The computation time of the convex OCP (17) is investigated with fixed weights $\left(W_{1}, W_{2}\right)$ of the objective (??) and for different numbers of vehicles crossing at $\mathrm{CZ}$, ranging 
from $N=10$ to $N=100$ at a fixed arrival rate of $1000 \mathrm{veh} / \mathrm{h}$. The resulting computation times are reported in Table II. As it can been seen, the optimal solution in each case can

TABLE II

COMPUTATIONAL TIME OF THE CONVEX OCP (17) FOR DIFFERENT $N$ UNDER ARRIVAL RATE OF $1000 \mathrm{VEH} /$ HOUR.

\begin{tabular}{l|ccccc}
\hline \hline Number of CAVs, $N$ & 10 & 20 & 30 & 50 & 100 \\
\hline Computation time $[\mathrm{s}]$ & 1.03 & 1.74 & 2.17 & 3.28 & 5.89 \\
\hline \hline
\end{tabular}

be efficiently solved and the time required for computation is much smaller than the total travel time; see for example Fig. 5. To verify that the FIFO policy and safety constraints are followed during the simulations, the traveled distance of each CAV is plotted against time in Fig. 5 for the case with $N=20$, as an example. The profiles illustrated in Fig. 5

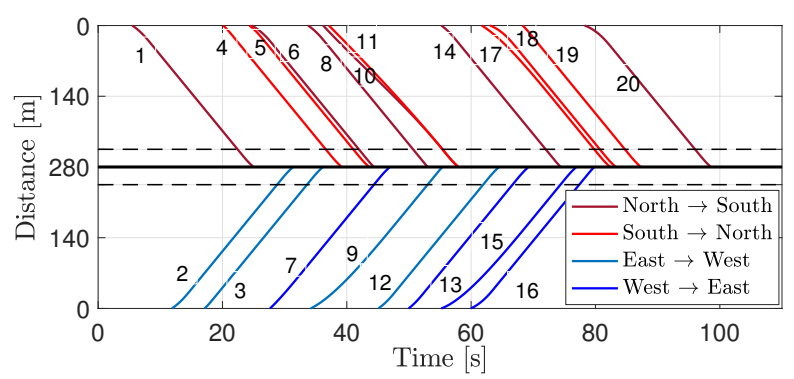

Fig. 5. Traveled distance trajectories of CAVs by solving the OCP (17) with $N=20 \mathrm{CAVs}$ at arrival rate of $1000 \mathrm{veh} / \mathrm{h}$. The two dashed lines and the solid line correspond to the entrance and exit of the MZ, respectively.

verify that the FIFO policy and both rear-end (5) and lateral (6) safety constraints are followed throughout the simulation, and therefore, the validity of the convex solution for the original problem is verified.

Next, the optimal solutions for various combination of weighting factors (under the same initial conditions) are investigated to study the trade-off between travel time and energy consumption. In Fig. 6, the Pareto fronts for four different arrival rates and fixed number of vehicles, $N=20$, are shown. The results indicate that for all cases an increase

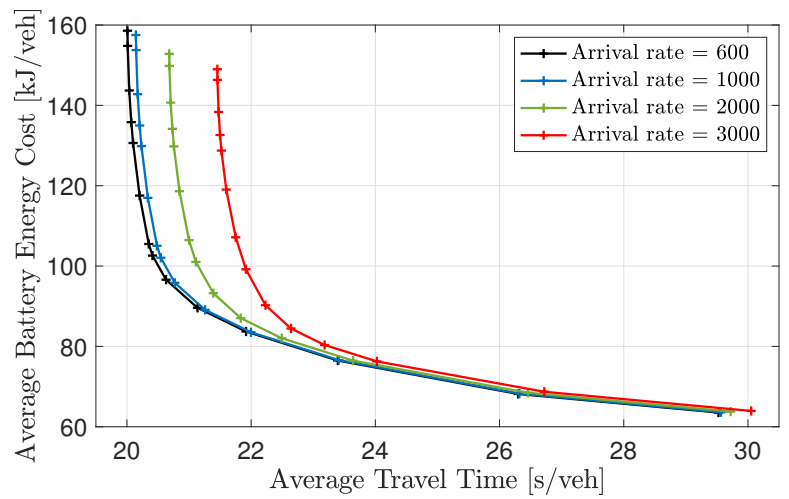

Fig. 6. Trade-offs between average battery energy cost and average travel time per vehicle for arrival rate from $600 \mathrm{veh} / \mathrm{h}$ to $3000 \mathrm{veh} / \mathrm{h}$.

in travel time of $20 \%$ can lead to a roughly $50 \%$ reduction in fuel consumption, while further increase in travel time can eventually lead to up to $62 \%$ fuel consumption reduction.
These results highlight the importance of examining the fuel consumption-travel time trade-off, as small compromises in the travel time produce significant fuel savings.

By comparing the optimal solutions for different arrival rates, it follows that the optimality deteriorates as the arrival rate increases. The reason is that a high traffic intensity brings more restriction on the motion of CAVs, and tends to yield a constrained solution, which is less optimal than an unconstrained one. Furthermore, the degradation becomes much more significant when the weight emphasizes more on the travel time than the energy usage, and when $W_{1} \gg W_{2}$ the reduction of average travel time is saturated by the upper limit of the velocity through $(S+L) / v_{\max }$ (that is $18.7 \mathrm{~s}$ in this example). Conversely, the energy usages for all four cases are close to each other when the energy outweighs the travel time, $W_{2} \gg W_{1}$.

For illustrative purposes, the optimal speed profiles for two representative cases are shown in Fig. 7. In the top two
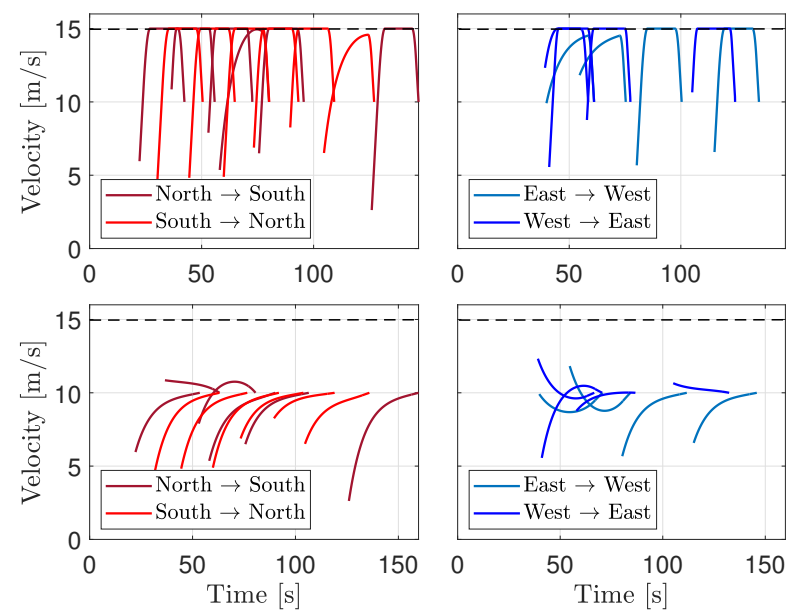

Fig. 7. Optimal speed profiles for all CAVs at an arrival rate of $1000 \mathrm{veh} / \mathrm{h}$ with $W_{1} \gg W_{2}$ (top) and $W_{1} \ll W_{2}$ (bottom). The speed limit $v_{\max }$ is shown by dash lines.

figures, with much higher weight on the travel time than the energy, most vehicles accelerate to $v_{\max }$ and cruise at this speed until they approach the exit of the MZ, at which point they slow down to $10 \mathrm{~m} / \mathrm{s}$ to satisfy the boundary condition. The heavy braking at the end allows substantial energy recovery, which can reduce the total energy consumption. When the weights emphasize more on the battery usage, the differences in the optimal solutions of different weight cases become negligible as all CAVs have sufficient time to drive efficiently, as shown in the bottom two figures of Fig. 7. Finally, by reference to Fig. 6, it has been found that further decrease in the arrival rate below 600 makes a negligible impact on the optimality as the traffic intensity is low enough to allow each CAV to follow the unconstrained optimal solutions.

To verify the benefit of removing the conservative assumption that presumes a constant cruising speed for all CAVs over the MZ, we compare the solutions of two optimization frameworks: 1) proposed scheme with non-constrained velocity within $\mathrm{MZ}$ and $v_{i}(L+S)=\bar{v}$, and 2) scheme in [11] with constrained velocity within $\mathrm{MZ} v_{i}(s)=\bar{v}, s \in$ $[L, L+S]$. In case 1$)$, the optimization is solved over the 
full horizon $[0, L+S]$ and the energy and time consumption are obtained straightforwardly. In contrast, the energy and time consumption for case 2) are obtained by adding up the results from the optimization over $[0, L]$ and the energy and time required to pass through the $\mathrm{MZ}$ at $\bar{v}$. For the sake of fair comparison, the same initial conditions are employed in both cases. The comparative results are presented in Fig. 8. As it can be seen, the removal of the assumption can enhance

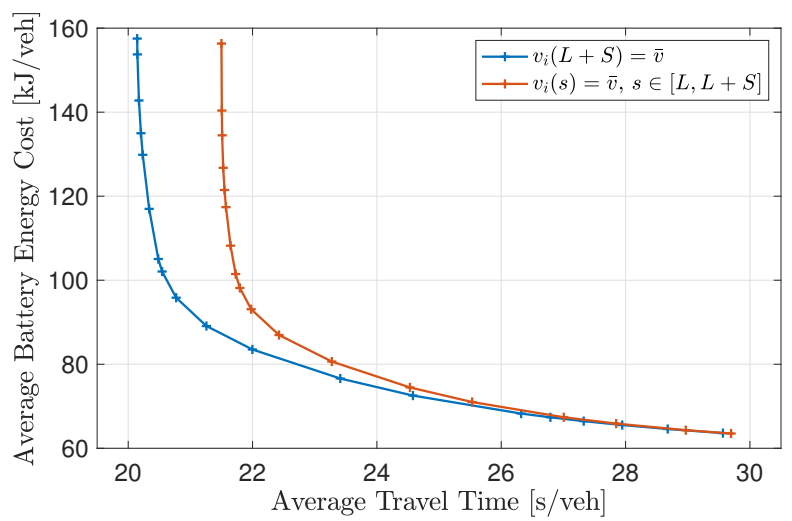

Fig. 8. Trade-offs of solving OCP (17) for two constraints cases: a) $v_{i}(L+$ $S)=\bar{v}$, and b) $v_{i}(s)=\bar{v}, s \in[L, L+S]$, at $1000 \mathrm{veh} / \mathrm{h}$ arrival rate.

the optimality because of the extended optimization horizon.

Finally, it is important to verify that the equality condition of (11b) holds at all times. A representative case with $W_{1} \ll$ $W_{2}$ shows this to be the case in Fig. 9. This example further

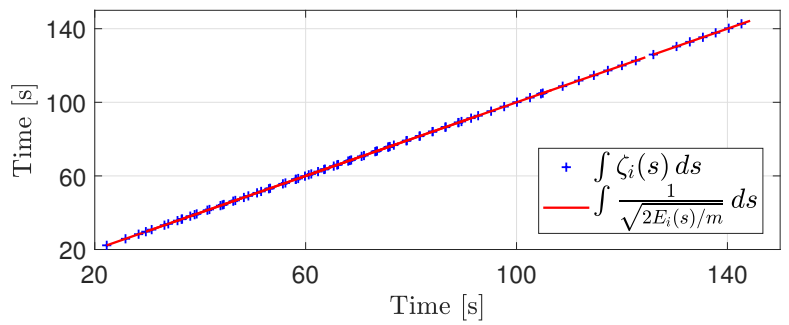

Fig. 9. The integration of $\zeta_{i}(s)$ and $1 / \sqrt{2 E_{i}(s) / m}$ from an example solution of the convex OCP with $W_{1} \ll W_{2}$.

implies the validity of the formulation, as further emphasis on the time consumption (an increase in $W_{1}$ ) can only reinforce the equality condition (11b).

\section{COnCLusions}

The problem of optimally controlling CAVs crossing a signal-free intersection is addressed in this paper. With the popularization of electrified vehicles, all the CAVs are assumed to be battery electric vehicles. The control objective is to minimize a weighted sum of the aggregate electric energy consumption and traveling time of all CAVs subject to safety constraints and powertrain operational limits. By utilizing convex modeling techniques, the overall optimal control problem is formulated as a convex optimization problem, which can be efficiently solved. The numerical examples investigate the trade-off between travel time and energy consumption for different traffic conditions, characterized by the arrival rates of the intersection. Moreover, the conservativeness of the convex OCP formulation and the validity of the optimal solution are also verified.
Future work consists in developing a robust optimization scheme (e.g., robust MPC), where uncertainties in initial conditions (arrival time and speed) and the impact of communication delays and noise can be addressed. Moreover, the optimality can be further enhanced by removing the FIFO policy to enable global scheduling of all CAVs.

\section{REFERENCES}

[1] J. Guanetti, Y. Kim, and F. Borrelli, "Control of connected and automated vehicles: State of the art and future challenges," Annual reviews in control, vol. 45, pp. 18-40, 2018.

[2] H. Yang, H. A. Rakha, and M. V. Ala, "Eco-cooperative adaptive cruise control at signalized intersections considering queue effects," IEEE Transactions on Intelligent Transportation Systems, vol. 18, no. 1, pp. 1575-1585, 2017

[3] Z. Wang, G. Wu, P. Hao, and M. J. Barth, "Cluster-wise cooperative eco-approach and departure application along signalized arterials,' in IEEE 20th International Conference on Intelligent Transportation Systems. IEEE, 2017.

[4] Y. Bian, Y. Zheng, W. Ren, S. E. Li, J. Wang, and K. Li, "Reducing time headway for platooning of connected vehicles via v2v communication," Transportation Research Part C: Emerging Technologies, vol. 102, pp. 87-105, 2019.

[5] M. Choi, A. Rubenecia, and H. H. Choi, "Reservation-based traffic management for autonomous intersection crossing," International Journal of Distributed Sensor Networks, vol. 15, no. 12, 2019.

[6] D. Cakija, L. Assirati, E. Ivanjko, and A. L. Cunha, "Autonomous intersection management: A short review," in 2019 International Symposium ELMAR. IEEE, 2019, pp. 21-26.

[7] E. Namazi, J. Li, and C. Lu, "Intelligent intersection management systems considering autonomous vehicles: A systematic literature review," IEEE Access, vol. 7, pp. 91 946-91 965, 2019.

[8] J. Rios-Torres and A. A. Malikopoulos, "A survey on the coordination of connected and automated vehicles at intersections and merging at highway on-ramps," IEEE Transactions on Intelligent Transportation Systems, vol. 18, no. 5, pp. 1066-1077, 2017.

[9] S. A. Fayazi and A. Vahidi, "Mixed-integer linear programming for optimal scheduling of autonomous vehicle intersection crossing," IEEE Transactions on Intelligent Vehicles, vol. 3, no. 3, pp. 287-299, 2018.

[10] A. Hadjigeorgiou and S. Timotheou, "Optimizing the trade-off between fuel consumption and travel time in an unsignalized autonomous intersection crossing," in 2019 IEEE Intelligent Transportation Systems Conference (ITSC). IEEE, 2019, pp. 2443-2448.

[11] B. Chen, X. Pan, S. A. Evangelou, and S. Timotheou, "Optimal control for connected and autonomous vehicles at signal-free intersections," in IFAC World Congress, 2020.

[12] K. Zhang, D. Zhang, A. de La Fortelle, X. Wu, and J. Gregoire, "State-driven priority scheduling mechanisms for driverless vehicles approaching intersections," IEEE Transactions on Intelligent Transportation Systems, vol. 16, no. 5, pp. 2487-2500, 2015.

[13] A. P. Chouhan and G. Banda, "Autonomous intersection management: A heuristic approach," IEEE Access, vol. 6, pp. 53 287-53 295, 2018

[14] X. Qian, J. Gregoire, A. De La Fortelle, and F. Moutarde, "Decentralized model predictive control for smooth coordination of automated vehicles at intersection," in 2015 European Control Conference (ECC). IEEE, 2015, pp. 3452-3458.

[15] A. A. Malikopoulos, C. G. Cassandras, and Y. J. Zhang, "A decentralized energy-optimal control framework for connected automated vehicles at signal-free intersections," Automatica, vol. 93, pp. 244256, 2018.

[16] S. Uebel, N. Murgovski, B. Bäker, and J. Sjöberg, "A two-level mpc for energy management including velocity control of hybrid electric vehicles," IEEE Transactions on Vehicular Technology, vol. 68, no. 6 , pp. 5494-5505, 2019.

[17] T. Liu and Selpi, "Comparison of car-following behavior in terms of safety indicators between china and sweden," IEEE Transactions on Intelligent Transportation Systems, 2019.

[18] B. Chen, S. A. Evangelou, and R. Lot, "Series hybrid electric vehicle simultaneous energy management and driving speed optimization," IEEE/ASME Transactions on Mechatronics, vol. 24, no. 6, pp. 27562767,2019

[19] D. R. Lopes and S. A. Evangelou, "Energy savings from an ecocooperative adaptive cruise control: a bev platoon investigation," 2019 18th European Control Conference (ECC), pp. 4160-4167, 2019.

[20] M. Grant and S. Boyd, "CVX: Matlab software for disciplined convex programming, version 2.1," http://cvxr.com/cvx, Mar. 2014. 